\title{
Simultaneous Cardiac and Neurological Monitoring to Assess Chemical Exposures and Drug Toxicity in Xenopus Laevis
}

Xing Xia ${ }^{1}$, Jimmy Zhang ${ }^{2}$, Manoj Vishwanath ${ }^{3}$, Sadaf Sarafan ${ }^{1}$, Ramses Seferino Trigo Torres$^{2}$, Tai Le ${ }^{2}$, Michael P.H. Lau ${ }^{4}$, Anh H. Nguyen ${ }^{1,4}$, Hung Cao ${ }^{1,2,4}$

\section{Affiliation}

${ }^{1}$ Department of Electrical Engineering and Computer Science, UC Irvine, Irvine, CA, 92697, USA.

${ }^{2}$ Department of Biomedical Engineering, UC Irvine, Irvine, CA, 92697, USA.

${ }^{3}$ Donald Bren School of Information and Computer Sciences, UC Irvine, CA 92697 , USA.

${ }^{4}$ Sensoriis., Inc, Edmonds, WA, 98026, USA.

\section{Correspondence}

Hung Cao, Ph.D.

Assistant Professor of Electrical and Biomedical Engineering

University of California, Irvine

Irvine, CA 92697

E-mail: hungcao@uci.edu 


\section{Abstract}

2 Simultaneous monitoring of electrocardiogram (ECG) and electroencephalogram (EEG)

3 under chemical exposure requires innovative engineering techniques that can capture

4 minute physiological changes in studied animal models. However, this is often

5 administered with a bulky system that may cause signal distortions and discomfort for

6 animals. We develop an integrated bioelectronic sensing system to provide simultaneous

7 ECG and EEG assessment in real-time under chemical exposure for Xenopus laevis. The

8 microelectrode array (MEA) membrane with integrated ECG and EEG sensing offers an

9 opportunity to achieve multichannel noninvasive electrophysiological monitoring with

10 favorable dimensions and spatial resolution. To validate the performance of our system,

11 we assessed the ECG and EEG of Xenopus under exposure of Pentylenetetrazol (PTZ),

12 an epilepsy-inducing drug. Effects of PTZ were detected with clear ECG and EEG

13 alterations, including frequent ictal and interictal EEG events, $30 \mathrm{~dB}$ average EEG

14 amplitude elevations, abnormal ECG morphology, and heart rate changes. Overall, our

15 Xenopus-based real-time electrophysiology monitoring system holds high potential for

16 many applications in drug screening and remote environmental toxicity monitoring.

Keywords: Xenopus Laevis; Simultaneous ECG and EEG Monitoring; Drug Toxicity 


\section{Introduction}

Pharmaceutical development involves a complicated process of biochemical assays and validation processes in animal models and humans before distribution to market (Barros et al. 2008; MacRae and Peterson 2015; Zon and Peterson 2005). Conventionally, mammalian models for preclinical toxicity, safety assessment, and side-effect screening needed to be determined first before experiments could be conducted. Large mammalian models, such as monkeys and dogs, were used as they possess comparable size and excellent gene similarity to humans. However, they were not always the ideal choice due to high cost, time consumption, and complications in conducting the screens (Denayer et al. 2014). Some mammalian animals, especially dogs and nonhuman primates, faced strong ethical issues for such preclinical tests (Baumans 2004). Hence, small mammalian animal models, such as rodents and rabbits, had been widely used. Although they shared a number of electrophysiological similarities to humans, there were some notable disparities. For instance, their heart rate $(\mathrm{HR})$ was several hundred beats per minute (bpm) while the respective value for humans was $\sim 60-70 \mathrm{bpm}$, questioning their suitability for studying the changes in cardiac electrophysiology under the influence of drugs. Recently, other models, such as zebrafish, had been explored, owing to their fecundity, morphological and physiological similarity to mammals, and the complexity of the circadian clock in relation to behavioral, sleep cycle, cellular and molecular responses. For example, in (Cao et al. 2014) and (Lee et al. 2020), ECG and EEG alterations were evaluated for cryo-injury assessment and epilepsy studies. Nonetheless, due to the small size of zebrafish, developing high spatial resolution electrophysiology recording devices for long term monitoring is still challenging. Furthermore, zebrafish do not possess lungs, 
which limits numerous studies involving the respiratory system, such as COVID-19. All these call for an alternative model that can facilitate drug screening and discovery.

Simultaneous EEG and ECG monitoring had been used to identify neurocardiac dysfunction in human and mouse models of epilepsy (Kendirli et al. 2014; Mishra et al. 2018). As EEG and ECG recording were commonly acquired from different areas on the body, simultaneous monitoring was often implemented separately using two different systems (Seitsonen et al. 2000). As a result, it was challenging to reduce the device size and bring comfort to test subjects. Alternatively, Xenopus laevis (X. laevis) has a unique body structure that could facilitate non-invasive recording of electrophysiological signals from the brain and heart. First, $X$. laevis' hairless and highly conductive skin alleviates the difficulties in obtaining high signal to noise ratio (SNR) and stable biopotentials. Second, anatomical features such as the favorable location of the heart, the absence of ribs, and the special structure of the transverse process on vertebrae allows easier acquisition of the posterior ECG than the anterior ECG, which enables the recording of ECG and EEG from the same side of Xenopus body. Third, the short distance between Xenopus heart and brain permits the implementation of electrodes on a single piece of flexible membrane to obtain ECG and EEG signals simultaneously. These advantages provide the opportunity to minimize the device size without sacrificing comfort and signal quality, promoting the use of Xenopus as an alternative model for drug screening studies.

Pentylenetetrazol (PTZ) is an antagonist of gamma-aminobutyric acid (GABA) on GABA receptors via the Tert Butylbicyclophosphorothioate site (Huang et al. 2001). The PTZ compound was used as a typical model for screening potential novel antiepileptic drugs and has become the approved drug (Löscher 2011). PTZ can penetrate through 
the plasma membrane, allowing PTZ to induce seizuresvia intracellular sites of action (Bloms-Funke et al. 1996). The EEG alterations caused by PTZ effects had been studied in zebrafish (Cho et al. 2017) and X. laevis tadpoles (Hewapathirane et al. 2008). Both works reported detections of high amplitude spikes, the criteria for epileptic events. In (Cho et al. 2017), 4 channel EEG was obtained from zebrafish by an EEG electrode array made with gold deposited on a flexible polyimide substrate. However, due to the limited size of zebrafish brain, the electrode size must be deliberated in order to guarantee high quality and localized EEG signals. In addition, careful immobilization and perfusion were needed during recordings as zebrafish and tadpoles cannot stay out of water for long time, increasing the complexity of obtaining EEG signals.

In this work, we developed a single flexible MEA with gold electrodes on a flexible polyimide film to simultaneously assess ECG and EEG in adult $X$. laevis. The acquired multichannel electrophysiological signals were amplified by a differential amplifier and digitized by an analog to digital converter for further data analysis. Using our system, EEG and ECG signals acquired from PTZ-induced seizures were simultaneous monitored, displaying distinguishable changes during ictal and interictal stages as well as their strong correlation over time. The system rapidly and accurately responded to phenotype changes, which offer potential and practicality to build compact wearable electrophysiology monitoring devices based on $X$. laevis for various biological studies and monitoring. 


\section{Materials and Methods}

\subsection{Animals}

All animal protocols were reviewed and approved by the Institutional Animal Care and Use Committee (IACUC) protocol (\#AUP-21-066, University of California, Irvine). Xenopus were maintained in separated aquaria with $21^{\circ} \mathrm{C}$ freshwater cycling. The aquaria were placed in a room with a 12 h:12 h light: dark cycle. 29 adult wild-type female Xenopus were used for this study. Due to the distinct differences in body sizes and weight between female and male $X$. laevis and the limited availability of male $X$. laevis in our facility, only female Xenopus were utilized in the experiments. There should be no significant difference among male and female $X$. laevis subjects in terms of electrophysiological assessment.

\subsection{Microelectrode array (MEA) design and fabrication}

To ensure the biocompatibility and flexibility of the sensors for a uniform and secure contact with the skin of Xenopus, we chose to fabricate the electrodes on polyimide films with various thicknesses ranging from 25 to $125 \mu \mathrm{m}$ (Kapton, Dupont, Wilmington, DE, USA). First, a Au/Cr layer of $200 / 20 \mathrm{~nm}$ was deposited on the polyimide substrate Conventional lithography and wet etching processes were used to pattern the four 50$\mu \mathrm{m}$-diameter circular electrodes. After the cleaning and post baking process, the traces of the electrodes were encapsulated by a layer of hardened photoresist, with the electrode areas and contact pads exposed.

In compliance with the brain structure of a Xenopus, we positioned the 4-channel EEG working electrodes (WE) on the scalp above the right and left sides of the telencephalon and the mesencephalon (Fan et al. 2018) (Fig 1A). The reference 
electrode was located above the center of the cerebellum. Three total lengths $(25,35$, and $45 \mathrm{~mm}$ ) of MEAs were designed to cater to different demands of flexibility. The ECG working electrodes were integrated on the MEAs, which have a total length longer than $35 \mathrm{~mm}$. The distance between the ECG working electrode and the reference electrode was $20 \mathrm{~mm}$, referring to the average heart-brain distance of Xenopus we measured. The ECG and EEG recording electrodes share a common reference electrode. Since EEG signals have lower amplitudes than ECG signals, the baseline noises caused by brain signals from cerebellum area were filtered out by additional signal processing. The electrodes were led to contact pads with a size of $2 \mathrm{~mm} \times 0.35 \mathrm{~mm}$ each to fit Flat Flexible Cable (FFC) connectors. A subsidiary printed circuit board (PCB) was designed to facilitate better connections between the electrodes and the cables leading to the subsequent signal collecting system. On the PCB, a FFC connector (5034800, Molex, Lisle, IL) with a $0.5 \mathrm{~mm}$ pitch was routed to a 2 row, $2.54 \mathrm{~mm}$ female socket header connector. The fabricated flexible electrode membrane was easily slipped and locked into a FFC connector, and the signal transmitting cables were connected to the female sockets on PCB by male pin headers. The PCB with connected flexible MEAs were shown in Fig. 1B.

\section{[Figure 1]}

\subsection{ECG and EEG signal acquisition}

$X$. laevis subjects were deeply anesthetized by immersion in a buffered solution of $1.5 \mathrm{~g} / \mathrm{L}$ tricaine methane sulfonate (MS-222). The subjects were considered sufficiently anesthetized with the loss of toe pinch response. After anesthesia, the Xenopus was settled on a specially designed frog bed. The test bed was made with 
132 polydimethylsiloxane (PDMS), and possessed a concave surface to fit the shape and 133 flexibility of frogs' abdomens. Then, the MEA was placed dorsally based on the location

134 of brain and heart and fixed by a manual micromanipulator (M3301, World precision 135 instruments Inc., Sarasota, FL) to reduce vibrations and noises. Due to the different 136 shrinkages of the frog skin and the Kapton membrane, the electrodes automatically 137 adhered to the skin resulting in a better electrical connectivity. During the entire experiment, the anesthetized Xenopus and accompanying recording devices were placed 139 in a Faraday cage on a vibration-free table. The signals were amplified 10,000-fold by a

140

141

142

143

144 145 146

147

148

149

150

151

152

153

154

voltage differential amplifier (A-M Systems Inc. 1700 Differential Amplifier, Carlsberg, WA) and filtered between 1 and $500 \mathrm{~Hz}$ with a cut-off frequency of $60 \mathrm{~Hz}$ (notch). The filtered signals were then digitized at a sampling rate of $1,000 \mathrm{~Hz}$ using a Data Acquisition Hardware (National Instruments USB-6251 DAQ device, Austin TX, and LabVIEW), and stored in a laptop for further data analysis. The full system setup is sketched and shown in Fig. 1C.

\subsection{Drug exposure}

To validate the functionality of the system, we conducted PTZ-induced epilepsy and anti-epileptic drug tests using valproic acid (VPA). Due to the excellent absorbability of Xenopus skin, direct immersion into drug solvent was utilized to treat the animal. The Xenopus were placed into a container filled with $200 \mathrm{~mL}$ drug solvent, with their eyes and noses above water. The drugs were transported in passively. Compared with injection, this method took a longer duration for the drugs to take effect. 30-mM PTZ dissolved in $1.5 \mathrm{~g} / \mathrm{L}$ MS-222 was used for PTZ drug exposure. The Xenopus were immersed in PTZ solution for 15 minutes. For the VPA treatment, the Xenopus were immersed in 3-mM 
VPA solution for 1 hour. During the drug treatment, the containers were covered by lids to prevent the rare case of Xenopus escaping the containers. After recordings, Xenopus were moved to a tank with flushing water to help them recover from anesthesia and drug effects. It took up to 30 minutes for Xenopus to fully recover. The drug-treated Xenopus were separated and maintained in recirculating tanks for 7 days.

\subsection{Data processing}

The ECG signal consisted of certain characteristics such as the P wave, PR interval, QRS complex, ST segment, T wave, and QT interval. For accurate detection of these characteristics, clear ECG signal was required. Therefore, noise cancellation was critical and essential. The lowpass filter and Savitzky-Golay finite impulse response (FIR) smoothing filter (SG filter) were used to suppress the powerline interference noise and remove the baseline wander. The baseline drifted due to respiration and could be normalized by using the lowpass filter. The SG filter proposed by Savitzky and Golay (Awal et al. 2011; Savitzky and Golay 1964) was based on local least-squares polynomial approximation; and it was demonstrated as one of the most popular noise cancellation and smoothing methods.

The obtained EEG signals were filtered using a $6^{\text {th }}$ order Butterworth bandpass filter from $0.5-40 \mathrm{~Hz}$. The selection of this frequency range was based on the frequency bands of interest, which includes delta $(0.5-4 \mathrm{~Hz})$, theta $(4-8 \mathrm{~Hz})$, alpha $(8-12 \mathrm{~Hz})$, beta $(12-30 \mathrm{~Hz})$ and gamma $(>30 \mathrm{~Hz})$. The Fourier transform (FFT) of the filtered signal was then calculated and visually compared between different groups to analyze the difference in frequency compositions of the signals. 


\section{Results}

\subsection{Xenopus EEG and ECG recording}

Five animals were used for device validation and then $24 X$. laevis were divided into 4 groups for the drug-induced epilepsy study. Each Xenopus frog was treated with drugs, and performed their 4-channel EEG and ECG recording were obtained 3 separate times. After each experiment, Xenopus were returned to circulating fresh water and allowed to recover from drug or anesthesia effects for at least 2 days. Xenopus in different groups were raised in different aquaria. Each recording lasted 20-30 minutes, depending on the duration that Xenopus were in anesthesia. It took approximately 12 minutes for Xenopus to be fully anesthetized in $1.5 \mathrm{~g} / \mathrm{L}$ MS-222 solution. Whenever the Xenopus lost toe-pinch reactions, we transferred the anesthetized Xenopus from the anesthesia solution to the frog bed. Adjusting the position of the Xenopus and adjusting the position of the MEA membrane took 2-3 minutes. Three different lengths $(25 \mathrm{~mm}, 35 \mathrm{~mm}, 45 \mathrm{~mm})$ and 3 different thicknesses $(25 \mu \mathrm{m}, 75 \mu \mathrm{m}, 125 \mu \mathrm{m})$ of the polyimide membrane were tested. The $75-\mu \mathrm{m}$ thick, $25-\mathrm{mm}$ long probe and the $125-\mu \mathrm{m}$ thick, $35-\mathrm{mm}$ and $45-\mathrm{mm}$ long probes displayed the best performances. These probes had similar flexibility and strength to the skin of Xenopus; thus, the conformability and longevity were significantly stronger, especially for long term recordings. In some of the recordings, we found that the EEG signals were noisy if the Xenopus scalp had too much mucus. We used paper towels to gently wipe the area before applying electrodes, greatly improving the signal quality. The use of a vibration-free table and a Faraday cage also contributed to obtain a favorable signal-to-noise ratio (SNR) (Defreitas et al. 2012; Tran et al. 2019). 
signals were obtained from the electrodes placed near the left and right sides of the telencephalon (LT and RT) and the left and right sides of the mesencephalon (LM and RM). This segment of recording included 20 seconds of normal EEG (baseline) followed by 20 seconds of the EEG during seizures of epilepsy. Periodic sharp spikes with much higher amplitude than the baseline EEG were observed and defined as the criteria for ictal seizures. More detailed EEG during seizures is shown in Fig. 2B. The signals from the left and right sides of the brain were similar in patterns and amplitudes, but comparing the signals from the telencephalon and the mesencephalon displayed numerous differences. Some spikes were clear in the LM and RM channels but difficult to see in LT and RT channels. Some high spikes were contrary in position, implying the origin of these abnormal electrical signals may be from the midbrain area (Saccomanno et al. 2020; Truszkowski et al. 2016). Fig. $2 \mathrm{C}$ shows the typical patterns of EEG before and after each seizure. There were periods of signals that had much higher amplitude spikes than regular EEG. We identified these spikes with $200-600 \mu \mathrm{V}$ high amplitudes as an ictal event. In the 20 seconds of recording shown in Fig. $\mathbf{2 C}$, there were two ictal events. These high amplitude spikes appeared 3-5 times in one second, and each ictal event contains 5-10 occurrences of such spikes, consistent through the whole recording. Between the two ictal events, some periodic bumps with lower amplitude and less sharpness were detected less frequently. These bumps were identified as an interictal event. The interictal events only appeared after the first appearance of the ictal event, and they disappeared before the ictal events ended. The small spikes of interictal intervals were around $30 \mu \mathrm{V}$, occurring 1-2 times in one second. The ictal and interictal events were not detected in the control group due to absence of focal epilepsy (Zijlmans et al. 2011). 


\section{[Figure 2]}

The simultaneous ECG signals were recorded by the working electrodes that were dorsally placed close to the heart. We also attempted to obtain ECG signals from the front chest of the Xenopus. Because of the body structure of Xenopus, it did not exhibit as high of a SNR as the ECG obtained posteriorly (Levis 2015). As a result, the ECG recording electrode was placed close to the EEG electrodes. decreasing the size of the MEA membrane to less than $2 \mathrm{~cm} \times 0.5 \mathrm{~cm}$ while maintaining high quality ECG and EEG. This result strongly attests to the benefit of using Xenopus as the model for ECG and EEG simultaneous monitoring. To minimize the size of the device and maximize the comfort the animals while monitoring, we chose to use the same reference electrode for both EEG and ECG that was placed on the cerebellum of Xenopus. Due to the short distance between heart and brain of the Xenopus, ECG and EEG signals from cerebellum were obtained with the same set of electrodes (Devuyst et al. 2008; Tong et al. 2001). Despite obtaining mixed ECG and EEG signals from the same channel, the recorded ECG signals were good enough to be used for morphological analysis. Fig. 3A shows an example of a portion of the raw signals recorded by the ECG electrodes. The R peaks had amplitudes around $250 \mu \mathrm{V}$, which were much higher compared with the baseline noises (less than $20 \mu \mathrm{V}$ ). The $\mathrm{R}$ peaks were definable without additional filtering, so the heart rate and $\mathrm{R}$ $R$ intervals could be detected from the raw signals manually. We also tried to record the ECG with an individual ECG reference electrode placed on the right leg of Xenopus. While it provided better ECG signals, the trade-off of the total sizes and the signal qualities made it less than ideal. Fig. 3B shows the ECG signals after noise cancellation and smoothing filtering. After data processing, the PQRST waves were detected clearly. The normalized 
ECG of Xenopus with PQRST marked is showed in Fig. 3C. We also obtained the human ECG and zebrafish ECG signals using the same system. Compared to human and zebrafish ECG, Xenopus ECG exhibited one extra wave between the $S$ and $T$ waves. This wave could be from PTZ-induced seizure for ECG change particularly in highsusceptibility frog individuals. ECG changes due to PTZ-induced seizure had not been well studied in Xenopus models; however, advantages in cardiac and brain systems of this model allow correlation of electrographic changes in the setting of drug-induced seizures to cardiac arrhythmia (Desai et al. 2017; Kuttab et al. 2019).

\section{[Figure 3]}

\subsection{PTZ-induced seizures and VPA anti-epileptic effects}

The ECG and EEG signals were recorded and analyzed from 4 groups of Xenopus. 12 Xenopus were divided into 4 groups with different drug treatments: 1) PTZ group, in which the Xenopus were treated with 30 mM PTZ mixed with MS-222; 2) VPA+PTZ group, in which they were immersed in $5 \mathrm{mM}$ VPA solution for 1 hour before they were transferred to the same PTZ treatment as the PTZ group; 3) VPA group, in which Xenopus were treated with $5 \mathrm{mM} \mathrm{VPA}$ for 1 hour; and 4) Control group, where no drug treatment was administered. Each Xenopus was anesthetized in $1.5 \mathrm{~g} / \mathrm{L} \mathrm{MS}-222$ and then recorded for more than 25 minutes.

The typical 1500-second-long recordings of raw EEG signals from the control group, PTZ group and the VPA+PTZ group are shown in Figs. 4A-4C, respectively. The 1500second-long samples were recorded by the LT working electrodes (channel 1), but the other 3 EEG channels showed similar results statistically, as all channels were able to 
record the same number of ictal events. The signals of the experimental groups were significantly different. The most distinguishable feature was the difference in the number of PTZ-induced seizures. EEG recorded in the control group did not show any occurrence of seizures. The average amplitude of control group EEG was $10 \mu \mathrm{V}$. For the PTZ group and the VPA+PTZ group, the ictal event occurrence, previously identified by spikes in amplitudes of $200-600 \mu \mathrm{V}$, was 34 times and 2 times, respectively. However, upon a closer look into the EEG patterns in the VPA+PTZ group, the epilepsy-like EEG spikes still existed and had lower amplitudes (Smith 2005a). The average amplitudes of the ictal spikes decreased $81.0 \%$ from $370 \mu \mathrm{V}$ to $70 \mu \mathrm{V}$. As a result, the criteria of an ictal event for $\mathrm{VPA}+\mathrm{PTZ}$ group was changed to $37.8-113.5 \mu \mathrm{V}$, proportional to the decrease in amplitudes of spikes. After recalculation, the ictal events in the VPA+PTZ group in the 1500 -second-long recording appeared 9 times, resulting in a $73.5 \%$ reduction in comparison with the PTZ group subject. These results proved that VPA can mitigate PTZinduced seizures in terms of occurrences and intensities which is in accordance with studies on other models (Puig-Lagunes et al. 2016; Romoli et al. 2019). The recordings obtained from different groups also validate our device for its capability and longevity to collect high SNR EEG for long term monitoring.

\section{[Figure 4]}

The Fast Fourier Transform (FFT) spectrums averaged across 4 recorded channels of the 4 groups are shown in Fig. 4D. It quantified the amount of different frequency components present in the given signal. In general, all 4 groups had higher amplitudes in low frequencies, which is a characteristic for all EEG signals. It is observable that the 
amplitude of the PTZ group was $30 \mathrm{~dB}$ higher than the control group and the VPA group for the entire frequency range of frequencies $(0-50 \mathrm{~Hz})$. The averaged amplitude of EEG signals in 4 groups over 200-second-long recordings was also calculated and compared. The Control group and the VPA group did not have noticeable differences, indicating that the VPA did not cause significant EEG changes in amplitudes. The results are shown in Fig. 4E. With the immersion of PTZ, the average EEG amplitude increased $833.9 \%$ in the control group. In the VPA treated groups, the amplitude only increased $70.0 \%$ after the immersion of PTZ, resulting in a $91.6 \%$ mitigation of PTZ effects. The averaged amplitude in the control group and VPA treated group did not show significant differences, which indicated that the VPA did not have mitigation effects on non-seizure EEG amplitude.

\subsection{Abnormal ECG and its features caused by PTZ}

In our experiments, the heart rates, R-R intervals, QTc intervals and morphological characteristics of Xenopus ECG were analyzed and compared. For the 6 Xenopus frogs in the PTZ group, we calculated the average heart rate every 2 minutes, from the first to the $29^{\text {th }}$ minute mark in the recording. Since the heart rate of Xenopus differed widely in certain individuals (from $11 \mathrm{bpm}$ to $52 \mathrm{bpm}$ in our dataset), the percentage change of heart rate was chosen as the parameter to be analyzed. The average heart rate of the whole 30 minutes recording was calculated individually. The relative heart rate (RHR), which is the heart rate at one moment in time divided by the averaged heart rate of the whole recording, was used to determine the relative variation of heart rate during the whole recording. The average HR variation of the 6 Xenopus after the treatment of PTZ were shown in Fig. 5A. To investigate the effect of seizures on the heart rate, we first determined the occurrences of epileptic seizures from simultaneously recorded EEG 
signals. The first appearance of a typical ictal event in the EEG signals was defined as the boundary of the preictal stage and the ictal stage (Fisher et al. 2014). The boundaries appeared at around 10 minutes into the 6 ECG recordings. The 2 stages were marked with different colors in Fig. 5A. By investigating the treads of the Xenopus heart rate variation in PTZ group, we found that the relative heart rate did not increase when seizures occurred. On the contrary, the heart rate was even lower in the Ictal stage than the Preictal stage for all the Xenopus tested. To study the anesthetic inhibiting effects on the heart rate, the control group was were introduced and compared. The control group consisted of Xenopus that were only treated with MS-222 at the same concentration and duration as other experimental groups. Fig. 5B showed the relative heart rate (RHR) variation in 30-minute-long recordings of the control group. The RHR curve of the PTZ group was shown in Fig. 5B as the red dotted line for comparison. Both groups had very similar heart rate variation from the 10th -30 th minutes of the recording, which was defined as Ictal stage in the PTZ group. But the RHR variation from the beginning to 10th minute in the control group recording was more moderate. This comparison indicated that the PTZ has caused increases in heart rate, but the heart rate gradually returned to normal. Additionally, the ictal events did not have direct relations to the heart rate.

\section{[Figure 5]}

PTZ also induced morphological changes on the ECG patterns (Akyuz et al. 2021; Ruby et al. 2008). The ECG morphology changes were not identical for all Xenopus frogs. From the PTZ group, one Xenopus was found to possess gradually inverted T waves during the recording. The ECG signals after PTZ treatment was shown in Fig. 6A. Four intervals of ECG were selected, which are (a), the start of the recording; (b), the end of 
336 the preictal period; (c), the start of the ictal events; and (d), the end of the recording. From

337 (a) to (d), the R-R intervals increased from 1.7 seconds to 4.7 seconds, and the T waves changed from upright in (a) and (b), to flat in (c), and then inverted in (d). As the figure depicts, the morphology of other waves did not have significant changes besides $T$ waves. Another Xenopus was detected to have 3 minutes of arrhythmia when the seizures happened. The ECG is shown in Fig. 6B. The arrhythmia happened right after the occurrence of the first ictal event. Before and after this 3-minute interval of arrhythmia, the R-R interval was around 2.8 seconds. When the arrythmia occurred, the heart beats were in pairs as one fast heart rhythm and one slow rhythm. The R-R intervals were 1.6 seconds and 2.7 seconds, respectively. In contrast, the morphology of ECG did not have noticeable changes for all subjects in the control group.

\section{[Figure 6]}

\section{Discussion}

\subsection{Simultaneous ECG and EEG monitoring}

Electrophysiological monitoring is important to biological and medical research. ECG and EEG recordings in humans have been widely used in clinical diagnostics and vital sign monitoring for decades. In recent years, as the development of fabrication technology and electrical engineering, small feature size sensors and electronics were applied into wearable and implantable devices. These efforts provided opportunities to implement high spatial resolution ECG and EEG monitoring on small animal models. Rats and mice were some of the most popular lab animal models. Many researchers presented wired or wireless multichannel rodent EEG recording devices (Choi et al. 2010; Pinnell et
al. 2016). Some works also included a simultaneous ECG (Mishra et al. 2018). ECG and 
EEG recordings on zebrafish were realized as well, but integrating them is still challenging. Many of them used flexible electrodes to reduce the size and discomfort. Nevertheless, most of electrophysiology recording systems were based on rodents or zebrafish. The other animal models were lack of explorations.

During the first part of our work, we tried to place the ECG electrodes on different locations to acquire the best signal. Initially, we considered the chest to be the best location, similar to most other animals. However, we did not obtain ECG signals with satisfying quality, probably because of their ventral fat. Surprisingly, the unique location of Xenopus heart enabled the recording of good ECG signals dorsally. This finding compelled us to combine the ECG and EEG recordings in one piece of sensor for simultaneous recording. Another advantage that the Xenopus offered was their lack of neck structure. This characteristic not only flattened their dorsum but also significantly decreased the distance between the heart and the brain. The special body structure of Xenopus enabled simultaneous ECG and EEG recordings by a set of electrodes fabricated on a small piece of flexible substrate, which offered the opportunity to further minimize the device size.

In our work, non-invasive electrophysiology was chosen. Due to the thin skin and fat layer between the measuring spots and the signal sources, we were able to obtain clear ECG and EEG signals. The drawback of the closed heart and brain was the intermixing of ECG and EEG signals, but it was resolved by signal processing. In the future, invasive electrophysiology with implanted electrodes may be an option as it may provide signals with higher quality and stability. 


\subsection{Cardiac and cerebral influences caused by chemical induced epilepsy}

EEG is the most common diagnostic indicator for epilepsy diagnosis. During seizures, abnormal brain waves occur in EEG signals, often manifested as slow wave discharge around $3 \mathrm{~Hz}$ (Smith 2005b). High-density EEG was required to precisely determine the affected brain sections and the origins of seizures. However, in small animal models, normal electrodes did not provide enough spatial resolution. Thus, microelectrode arrays needed to be designed and fabricated. In our results, clear slow wave spikes were detected in all channels. It was noticeable that the signals from left mesencephalon were usually the highest in amplitude during seizures. This result indicated the origin of the discharges.

Even though researchers had determined that seizures originated from certain parts of the brain, the cardiac effects of seizures were also widely studied (Bloms-Funke et al. 1996; Huang et al. 2001; Nei 2009). In many clinical observations, the ictal events were linked to an increase of heart rates and many morphological changes (Eggleston et al. 2014). Chemical toxins were possible factors that have been reported to causes epilepsy, seizure or acute clinical syndromes (Jett 2012). The chemicals were toxic to the nervous system by causing hyperactive neurotransmission generated by a temporary change in the electrical functioning of the brain. In seizures, the brain's electrical rhythms were disrupted by sudden and synchronized bursts of electrical energy which tend to become imbalanced, resulting in alterations of the heart rate and abnormal rhythm (Nei 2009). Thus, we theorized that there was a possible connection between cardiac associated sudden unexpected death and epilepsy. Although the seizure-associated cardiac arrhythmias, such as ictal asystole that causes syncope, have been discovered, the 
404 overall risk of seizures on cardiac status still remained elusive. Recently, ECG and blood pressure results associated with PTZ-induced seizures and epilepsy were frequently encountered in elevated catecholamine release, which induce abnormal ECG (Akyuz et al. 2021; Ghosh et al. 2009; Nass et al. 2019; Zavala-Tecuapetla et al. 2020). Unlike most reported human heart rate changes, our results showed that the PTZ induced seizures did not cause an increase of heart rate while ictal events happened. This observation was consistent with a previous report (Randall and Rohse 1956), but contradictory to other reports in terms of PTZ effects (Auerbach et al. 2013; Beig et al. 2009). Since our experiments were done with Xenopus in anesthesia, the effects of anesthetic drugs should be investigated. Even though we had a control group to investigate the inhibition effects of MS-222 to heart rate variation, the drug-to-drug interactions have not been elucidated. Hence, there is a demand for awake electrophysiology monitoring in drug screening studies. Due to the limited number of Xenopus inspected, there were no general conclusions on how the PTZ influenced the heart and ECG. As many recorded Xenopus ECG signals exhibited abnormalities, PTZ induced negative effects on Xenopus hearts, but the specific influenced locations and mechanism remain uncertain.

\subsection{Pros and cons of using adult Xenopus in drug screenings}

Xenopus is a popular animal model, especially for developmental biology studies.

While adult Xenopus were not as widely used in research as their embryonic counterparts in recent decades, previous adult Xenopus studies have sequenced the complete genome and developed many transgenic models, bolstering the adult model for future drug screening studies. As discussed previously, small animal models possessed many advantages for drug screenings, such as lower costs and easier manipulation. However, 
427 small mammalian animals often have high heart rate, increase the difficulties and uncertainties for monitoring ECG alterations caused by drugs. Besides, higher heart rates also led to fast drug absorption and dissipation in body. These concerns encouraged researchers to find alternative models. Zebrafish was chosen by many researchers. However, due to their small size, many challenges were still encountered. $X$. laevis had a similar body size to rats, but their heart rate was much slower (11-52 in our monitoring), giving researchers more possibilities to look further into the morphological changes caused by drugs or chemicals. $X$. laevis is a leading model for regeneration research. In (Marshall et al. 2019), stage-dependent cardiac regeneration in Xenopus was discussed. researchers to determine the origin of heart regeneration ability. Additionally, Xenopus are aquatic animals, allowing for the intake of drugs by cutaneous respiration for easier facilitation of drug screening studies. With high absorbability skins of Xenopus, drug application approaches were not limited to injections. The flattened and streamlined body of Xenopus also eased the development of wearable or implantable monitoring devices. Furthermore, Xenopus are relatively inactive, eliminating the influences of high frequency movements, including motion noises and heart rate changes.

Despite their numerous advantages, there are some disadvantages of using adult $X$. laevis. Firstly, there are currently no standards of Xenopus ECG patterns. The threechambered Xenopus heart differs from the four-chambered human heart. As a result, the species-specific ECG differences need to be investigated to be translated to human 448 models. Second, their 1-2 year long generation time slows the transgenic lines production 449 (Beck and Slack 2001), which constrains reproduction and genetics studies. Third, due 
450 to the fully aquatic life habit, more cautions and concerns are needed for wearable and 451 implantable device development.

452

454

455

456

457

458

459

460

461

462

463

464

465

466

467

468

469

\section{Conclusion and Future Work}

In this work, a multichannel ECG-EEG recording system on Xenopus was developed. Although, $X$. laevis has not been widely used for studies involving electrophysiological monitoring, we discovered great potential in using this animal model for environmental monitoring and drug screening. The flexible MEA membrane placed dorsally on Xenopus non-invasively recorded the ECG and EEG simultaneously when the frogs were under anesthesia. There were no difficulties in installing the device. Normally, the recordings started within one minute after the Xenopus was sedated after anesthesia. The PTZ and VPA drug tests validated the feasibility of this system in real time monitoring of ECG and EEG for associated neurological and cardiovascular conditions. Both signals displayed detectable and distinct characteristics during different stages of seizures. With excellent skin permeability and easy-to-obtain electrophysiological signals, Xenopus could serve as a desired model for sensing and monitoring of chemical exposure and environmental changes. Future work includes the development of a miniaturized system on a more flexible substrate, such as parylene $\mathrm{C}$, with embedded microelectronics and wireless communication, so that awake electrophysiology signals can be monitored without interferences from Xenopus behavior. 


\section{Acknowledgement}

471 The authors would like to acknowledge the financial support from the NSF CAREER

472 Award \#1917105 (H.C.), the NSF \#1936519 (H.C), and the NIH SBIR grant

473 \#R44OD024874 (M.P.H.L and H.C.). We thank Lauren Schmiess-Heine and Roger

474 Geertsema for managing the Xenopus facility. 


\section{References}

Akyuz, E., Doganyigit, Z., Eroglu, E., Moscovicz, F., Merelli, A., Lazarowski, A., Auzmendi, J., 2021. Myocardial Iron Overload in an Experimental Model of Sudden Unexpected Death in Epilepsy. Frontiers in Neurology 12(4).

Auerbach, D.S., Jones, J., Clawson, B.C., Offord, J., Lenk, G.M., Ogiwara, I., Yamakawa, K., Meisler, M.H., Parent, J.M., Isom, L.L., 2013. Altered Cardiac Electrophysiology and SUDEP in a Model of Dravet Syndrome. PLOS ONE 8(10), e77843.

Awal, M.A., Mostafa, S.S., Ahmad, M., 2011. Performance analysis of Savitzky-Golay smoothing filter using ECG signal. International Journal of Computer and Information Technology 1(02), 24.

Barros, T., Alderton, W., Reynolds, H., Roach, A., Berghmans, S., 2008. Zebrafish: an emerging technology for in vivo pharmacological assessment to identify potential safety liabilities in early drug discovery. British journal of pharmacology 154(7), 1400-1413.

Baumans, V., 2004. Use of animals in experimental research: an ethical dilemma? Gene Therapy 11(1), S64-S66.

Beck, C.W., Slack, J.M., 2001. An amphibian with ambition: a new role for Xenopus in the 21st century. Genome Biol 2(10), REVIEWS1029-REVIEWS1029.

Beig, M.I., Chandra, R., Talwar, A., Fahim, M., Katyal, A., 2009. Epileptic seizure-induced hypertension and its prevention by calcium channel blockers: a real-time study in conscious telemetered rats. Canadian journal of physiology and pharmacology 87(7), $572-580$.

Bloms-Funke, P., Madeja, M., Musshoff, U., Speckmann, E.J., 1996. Effects of pentylenetetrazol on GABA receptors expressed in oocytes of Xenopus laevis: extra- and 
intracellular sites of action. Neuroscience letters 205(2), 115-118.

Cao, H., Yu, F., Zhao, Y., Zhang, X., Tai, J., Lee, J., Darehzereshki, A., Bersohn, M., Lien, C.-L., Chi, N.C., Tai, Y.-C., Hsiai, T.K., 2014. Wearable multi-channel microelectrode membranes for elucidating electrophysiological phenotypes of injured myocardium. Integrative Biology 6(8), 789-795.

Cho, S.-J., Byun, D., Nam, T.-S., Choi, S.-Y., Lee, B.-G., Kim, M.-K., Kim, S., 2017. Zebrafish as an animal model in epilepsy studies with multichannel EEG recordings. Scientific Reports 7(1), 3099.

Choi, J.H., Koch, K.P., Poppendieck, W., Lee, M., Shin, H.-S., 2010. High Resolution Electroencephalography in Freely Moving Mice. Journal of Neurophysiology 104(3), $1825-1834$.

Defreitas, J.M., Beck, T.W., Stock, M.S., 2012. Comparison of methods for removing electromagnetic noise from electromyographic signals. Physiological measurement 33(2), 147-158.

Denayer, T., Stöhr, T., Van Roy, M., 2014. Animal models in translational medicine: Validation and prediction. New Horizons in Translational Medicine 2(1), 5-11.

Desai, R., Rupareliya, C., Patel, U., Naqvi, S., Patel, S., Lunagariya, A., Mahuwala, Z., 2017. Burden of Arrhythmias in Epilepsy Patients: A Nationwide Inpatient Analysis of 1.4 Million Hospitalizations in the United States. Cureus 9(8), e1550-e1550.

Devuyst, S., Dutoit, T., Stenuit, P., Kerkhofs, M., Stanus, E., 2008. Cancelling ECG Artifacts in EEG Using a Modified Independent Component Analysis Approach. EURASIP Journal on Advances in Signal Processing 2008(1), 747325.

Eggleston, K.S., Olin, B.D., Fisher, R.S., 2014. Ictal tachycardia: The head-heart 
connection. Seizure 23(7), 496-505.

Fan, Y., Yue, X., Xue, F., Brauth, S.E., Tang, Y., Fang, G., 2018. The right thalamus may play an important role in anesthesia-awakening regulation in frogs. PeerJ 6, e4516.

Fisher, R.S., Scharfman, H.E., deCurtis, M., 2014. How can we identify ictal and interictal abnormal activity? Adv Exp Med Biol 813, 3-23.

Ghosh, A.K., Hatfield, E., Connolly, S., 2009. Catecholamine-induced transient myocardial dysfunction. BMJ Case Rep 2009, bcr12.2008.1349.

Hewapathirane, D.S., Dunfield, D., Yen, W., Chen, S., Haas, K., 2008. In vivo imaging of seizure activity in a novel developmental seizure model. Experimental Neurology 211(2), $480-488$.

Huang, R.Q., Bell-Horner, C.L., Dibas, M.I., Covey, D.F., Drewe, J.A., Dillon, G.H., 2001. Pentylenetetrazole-induced inhibition of recombinant gamma-aminobutyric acid type $A$ $(G A B A(A))$ receptors: mechanism and site of action. The Journal of pharmacology and experimental therapeutics 298(3), 986-995.

Jett, D.A., 2012. Chemical toxins that cause seizures. Neurotoxicology 33(6), 1473-1475. Kendirli, M.T., Aparci, M., Kendirli, N., Tekeli, H., Karaoglan, M., Senol, M.G., Togrol, E., 2014. Diagnostic Role of ECG Recording Simultaneously With EEG Testing. Clinical EEG and Neuroscience 46(3), 214-217.

Kuttab, H.I., Harris, E.A., Tataris, K.L., Tao, J., Beiser, D.G., 2019. Cardiac Arrhythmia Following an Epileptic Seizure. Clin Pract Cases Emerg Med 3(4), 354-356.

Lee, Y., Lee, K.J., Jang, J.-W., Lee, S.-i., Kim, S., 2020. An EEG system to detect brain signals from multiple adult zebrafish. Biosensors and Bioelectronics 164, 112315. Levis, J.T., 2015. ECG Diagnosis: Isolated Posterior Wall Myocardial Infarction. Perm J 
19(4), e143-e144.

Löscher, W., 2011. Critical review of current animal models of seizures and epilepsy used in the discovery and development of new antiepileptic drugs. Seizure 20(5), 359-368. MacRae, C.A., Peterson, R.T., 2015. Zebrafish as tools for drug discovery. Nature Reviews Drug Discovery 14(10), 721-731.

Marshall, L.N., Vivien, C.J., Girardot, F., Péricard, L., Scerbo, P., Palmier, K., Demeneix, B.A., Coen, L., 2019. Stage-dependent cardiac regeneration in \&lt;em\&gt;Xenopus\&lt;/em\&gt; is regulated by thyroid hormone availability. Proceedings of the National Academy of Sciences 116(9), 3614.

Mishra, V., Gautier, N.M., Glasscock, E., 2018. Simultaneous Video-EEG-ECG Monitoring to Identify Neurocardiac Dysfunction in Mouse Models of Epilepsy. Journal of visualized experiments : JoVE(131), 57300.

Nass, R.D., Hampel, K.G., Elger, C.E., Surges, R., 2019. Blood Pressure in Seizures and Epilepsy. Frontiers in Neurology 10(501).

Nei, M., 2009. Cardiac effects of seizures. Epilepsy Curr 9(4), 91-95.

Pinnell, R.C., Almajidy, R.K., Kirch, R.D., Cassel, J.C., Hofmann, U.G., 2016. A Wireless EEG Recording Method for Rat Use inside the Water Maze. PLOS ONE 11(2), e0147730. Puig-Lagunes, A.A., Manzo, J., Beltrán-Parrazal, L., Morgado-Valle, C., Toledo-Cárdenas, R., López-Meraz, M.-L., 2016. Pentylenetetrazole-induced seizures in developing rats prenatally exposed to valproic acid. PeerJ 4, e2709-e2709.

Randall, W.C., Rohse, W.G., 1956. The augmentor action of the sympathetic cardiac nerves. Circ Res 4(4), 470-475.

Romoli, M., Mazzocchetti, P., D'Alonzo, R., Siliquini, S., Rinaldi, V.E., Verrotti, A., 
567 Calabresi, P., Costa, C., 2019. Valproic Acid and Epilepsy: From Molecular Mechanisms

568 to Clinical Evidences. Curr Neuropharmacol 17(10), 926-946.

569 Ruby, N.F., Hwang, C.E., Wessells, C., Fernandez, F., Zhang, P., Sapolsky, R., Heller, 570 H.C., 2008. Hippocampal-dependent learning requires a functional circadian system. 571 Proceedings of the National Academy of Sciences of the United States of America 572 105(40), 15593-15598.

573 Saccomanno, V., Love, H., Sylvester, A., Li, W.-C., 2020. The early development and 574 physiology of \&lt;em\&gt;Xenopus laevis\&lt;/em\&gt; tadpole lateral line system. bioRxiv, $575 \quad 2020.2004 .2021 .052969$.

576 Savitzky, A., Golay, M.J., 1964. Smoothing and differentiation of data by simplified least 577 squares procedures. Analytical chemistry 36(8), 1627-1639.

578 Seitsonen, E., Yli-Hankala, A., Korttila, K., 2000. Are electrocardiogram electrodes 579 acceptable for electroencephalogram bispectral index monitoring? Acta 580 anaesthesiologica Scandinavica 44(10), 1266-1270.

581 Smith, S.J.M., 2005a. EEG in neurological conditions other than epilepsy: when does it 582 help, what does it add? Journal of Neurology, Neurosurgery \&amp;amp; Psychiatry 583 76(suppl 2), ii8.

584 Smith, S.J.M., 2005b. EEG in the diagnosis, classification, and management of patients 585 with epilepsy. Journal of Neurology, Neurosurgery \&amp;amp; Psychiatry 76(suppl 2), ii2. 586 Tong, S., Bezerianos, A., Paul, J., Zhu, Y., Thakor, N., 2001. Removal of ECG interference 587 from the EEG recordings in small animals using independent component analysis. 588 Journal of neuroscience methods 108(1), 11-17.

589 Tran, D., Dutoit, F., Najdenovska, E., Wallbridge, N., Plummer, C., Mazza, M., Raileanu, 
590 L.E., Camps, C., 2019. Electrophysiological assessment of plant status outside a Faraday 591 cage using supervised machine learning. Sci Rep 9(1), 17073-17073.

592 Truszkowski, T.L.S., James, E.J., Hasan, M., Wishard, T.J., Liu, Z., Pratt, K.G., Cline, H.T., 593 Aizenman, C.D., 2016. Fragile X mental retardation protein knockdown in the developing 594 Xenopus tadpole optic tectum results in enhanced feedforward inhibition and behavioral 595 deficits. Neural Development 11(1), 14.

596 Zavala-Tecuapetla, C., Cuellar-Herrera, M., Luna-Munguia, H., 2020. Insights into 597 Potential Targets for Therapeutic Intervention in Epilepsy. Int J Mol Sci 21(22), 8573.

598 Zijlmans, M., Jacobs, J., Kahn, Y.U., Zelmann, R., Dubeau, F., Gotman, J., 2011. Ictal and 599 interictal high frequency oscillations in patients with focal epilepsy. Clin Neurophysiol 600 122(4), 664-671.

601 Zon, L.I., Peterson, R.T., 2005. In vivo drug discovery in the zebrafish. Nature reviews 602 Drug discovery 4(1), 35-44. 

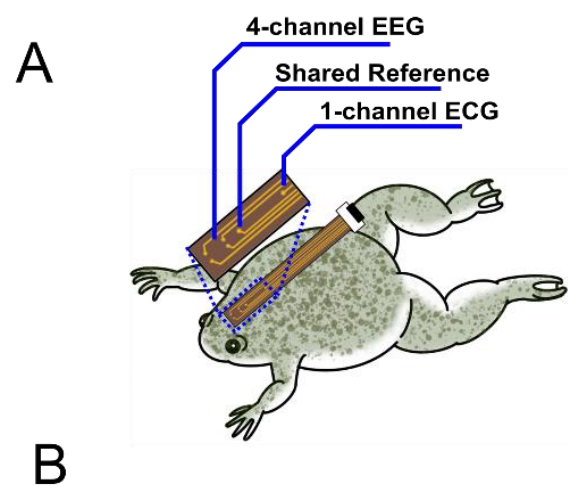

C

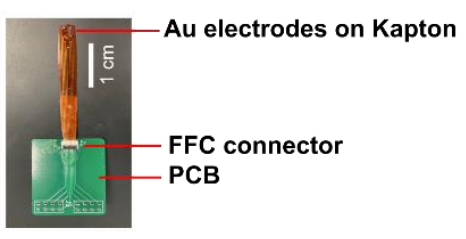

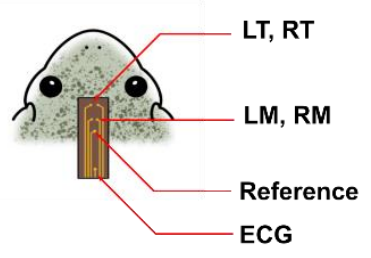

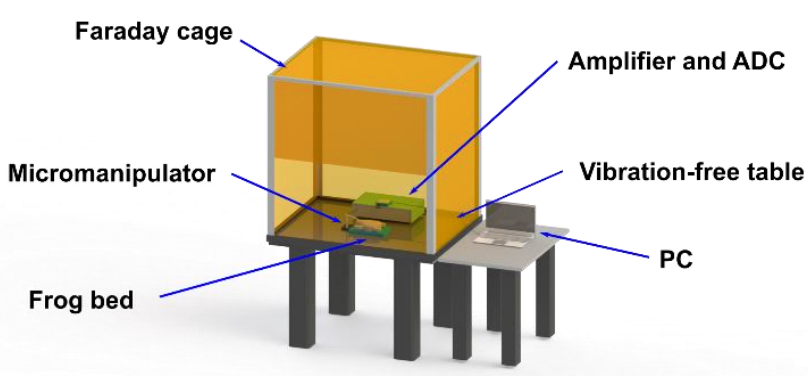

Fig.1. The experimental setup and devices to record EEG and ECG simultaneously.

A. 4 channel EEG and 1 channel ECG electrodes integrated on one polyimide membrane electrodes, left and right mesencephalon (LM and RM) electrodes, a reference electrode, 

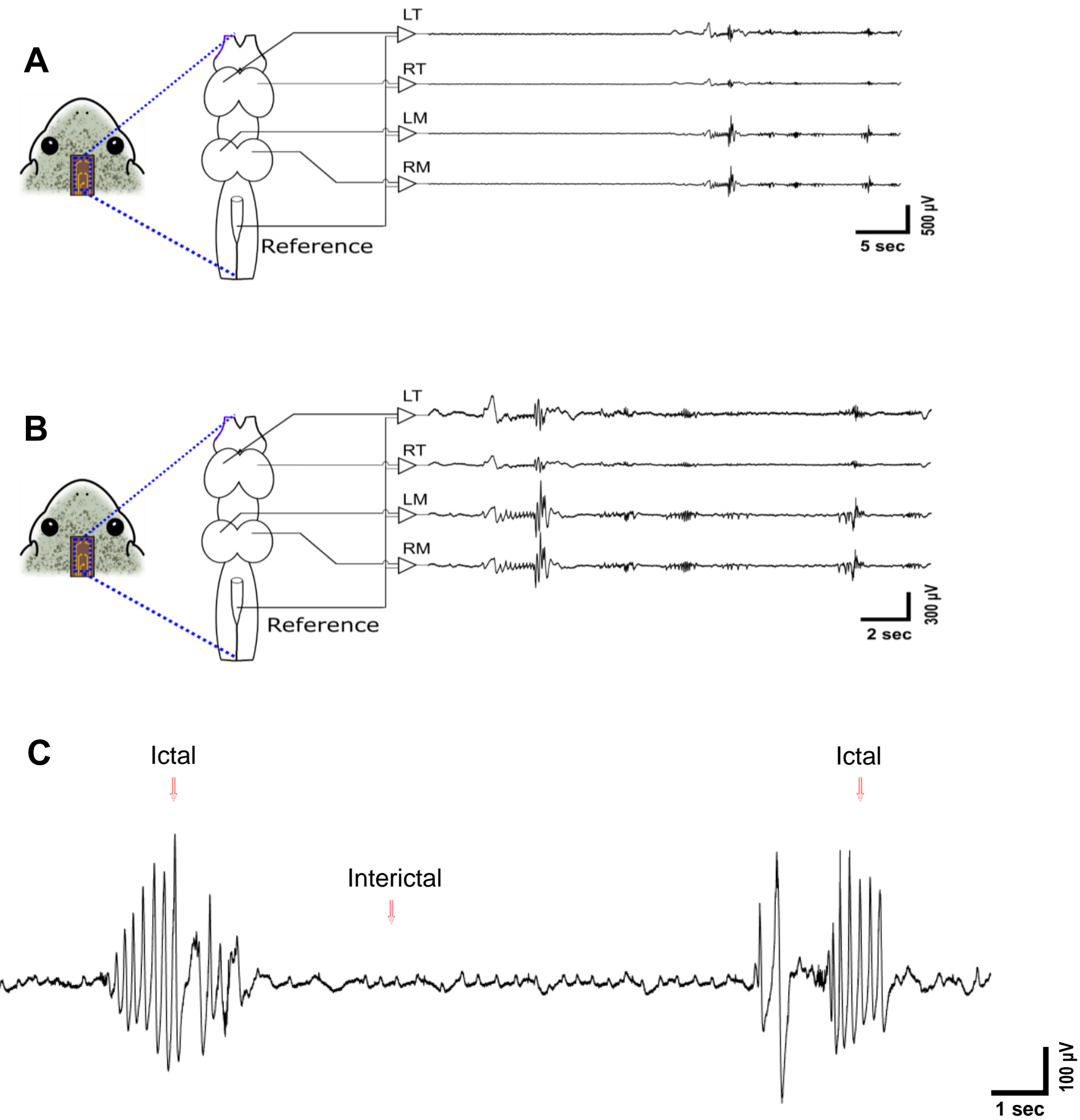

613 Fig. 2. Typical EEG signals recorded by the system. A. 4 channel EEG from different

614 locations of the brain in 40 seconds. B. 4 channel EEG when a seizure happened in 20

615 seconds. C. Typical EEG patterns of a seizure, includes two ictal events and one interictal 616 period. 
A

Raw ECG

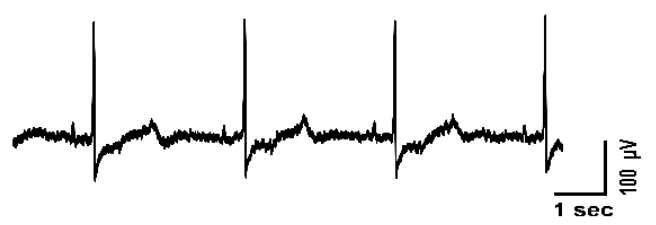

B

Filtered ECG

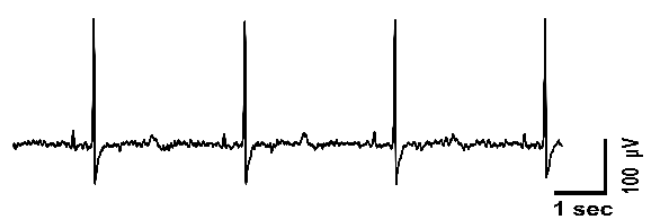

C

Xenopus QRS

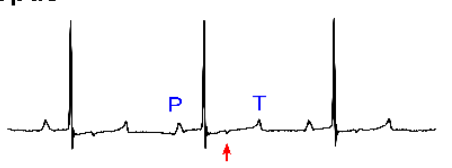

Zebrafish

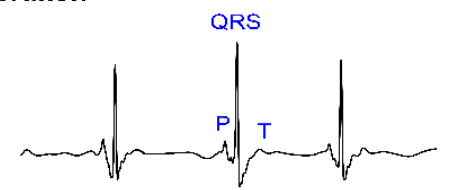

Human

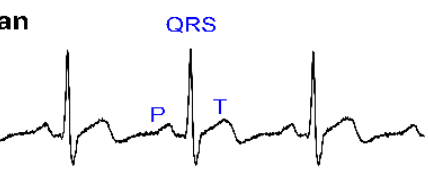

617

618

619

620

621

622

623

624 

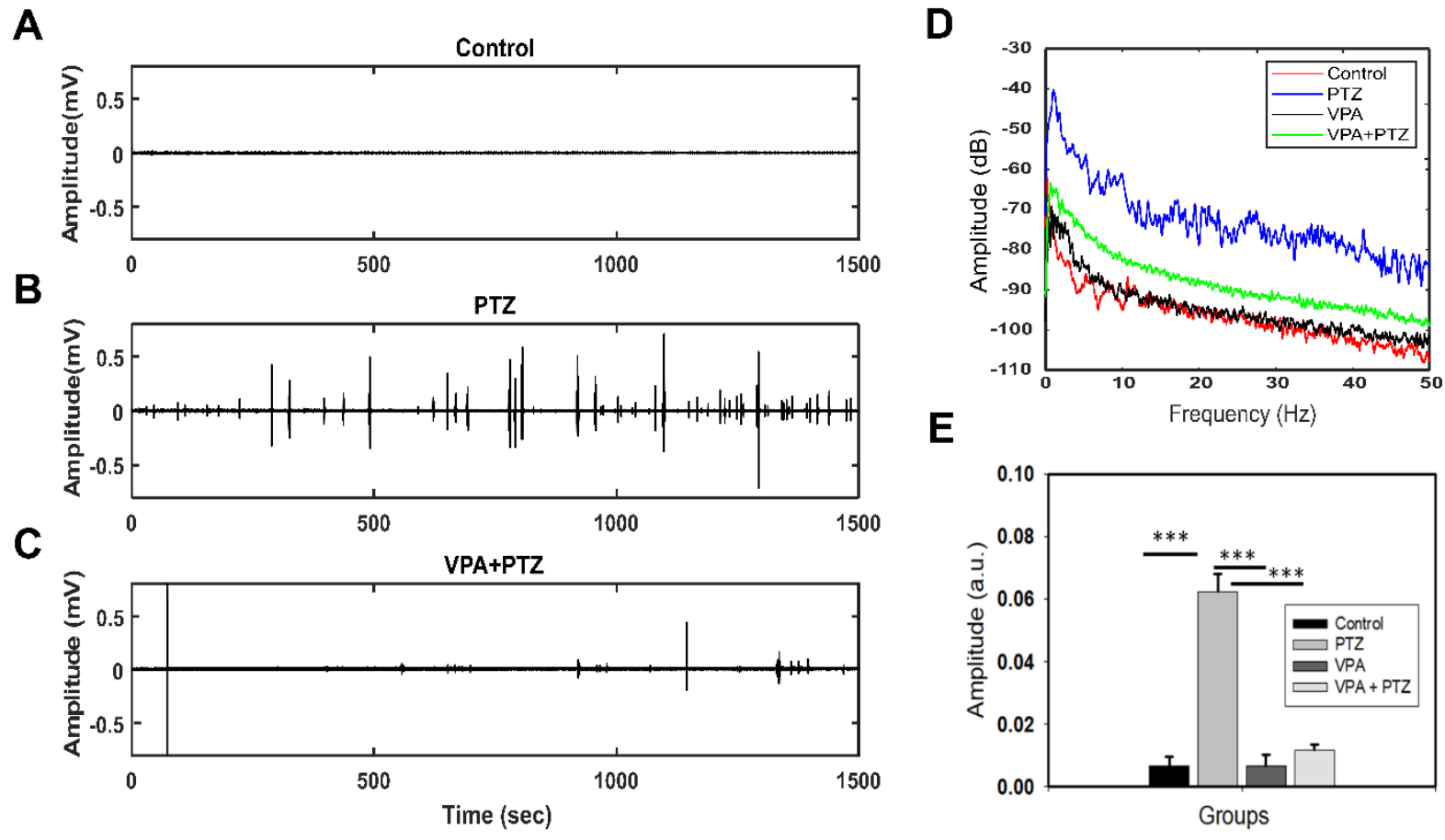

Fig. 4. PTZ and VPA effects to Xenopus EEG. A. 1500 second EEG of Xenopus in 627 control group; B. in PTZ group; C. in VPA+PTZ group. D. Fast Fourier transform of the 628 EEG in 4 groups. The groups treated with PTZ showed higher amplitude in $0-40 \mathrm{~Hz}$ 629 frequency domain, but the VPA inhibited the PTZ effects. E. The average amplitude of 630 EEG in 4 groups for quantified comparisons. 
A

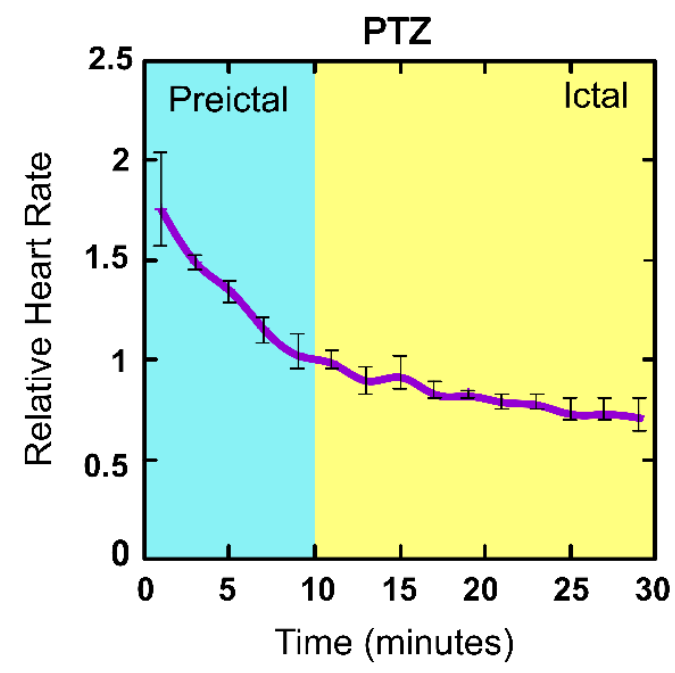

B

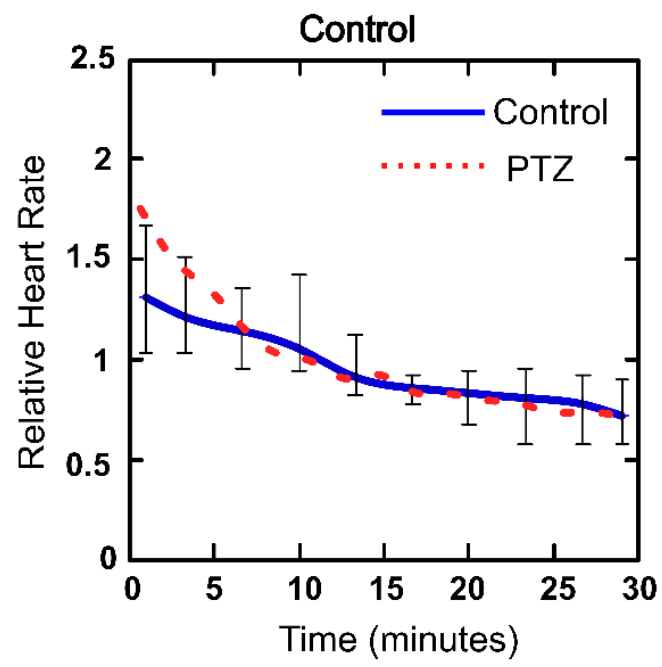

633 Fig. 5. Relative heart rate altering. A. The relative heart rate changings during the 30 634 minutes recordings in PTZ group. B. The comparison of relative heart rate trends in PTZ 635 and Control group. 
A

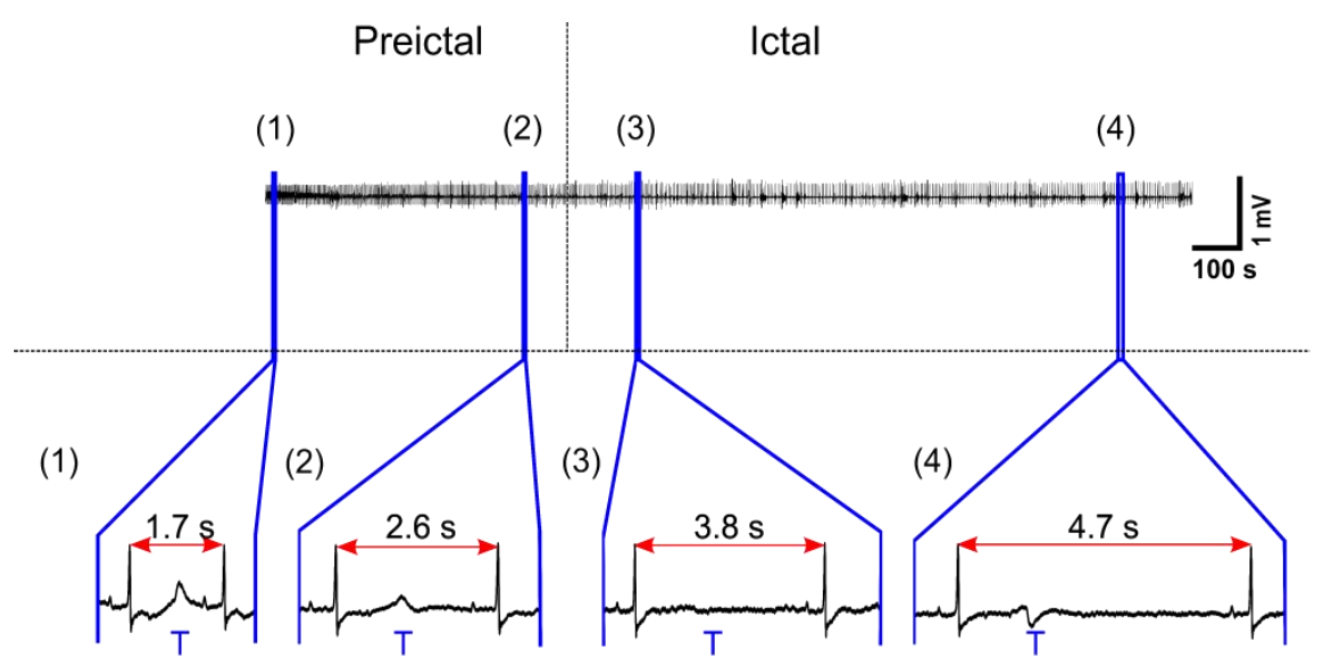

B
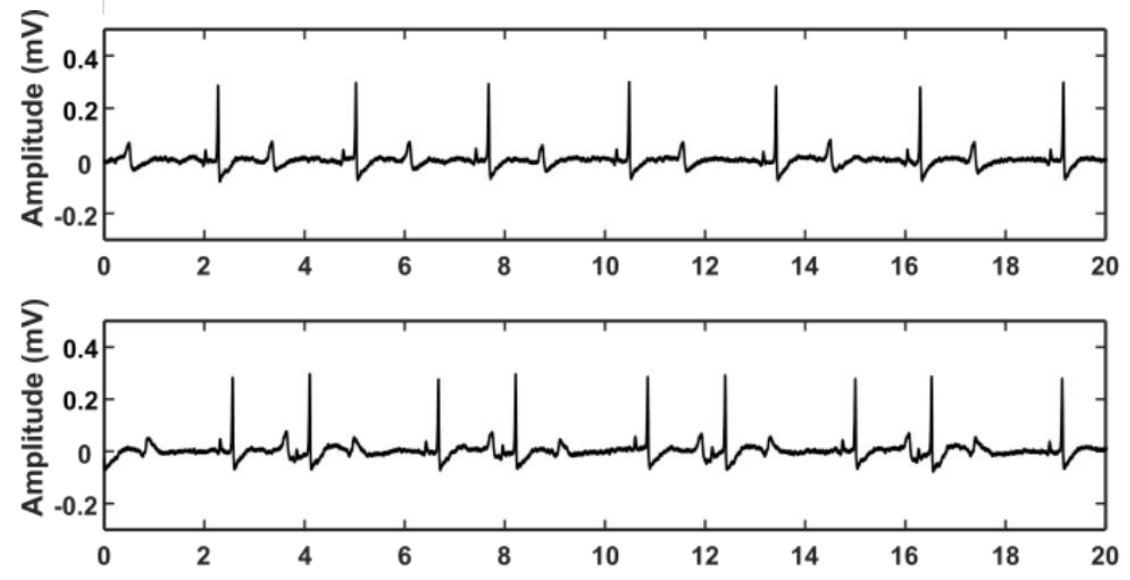

1st Ictal

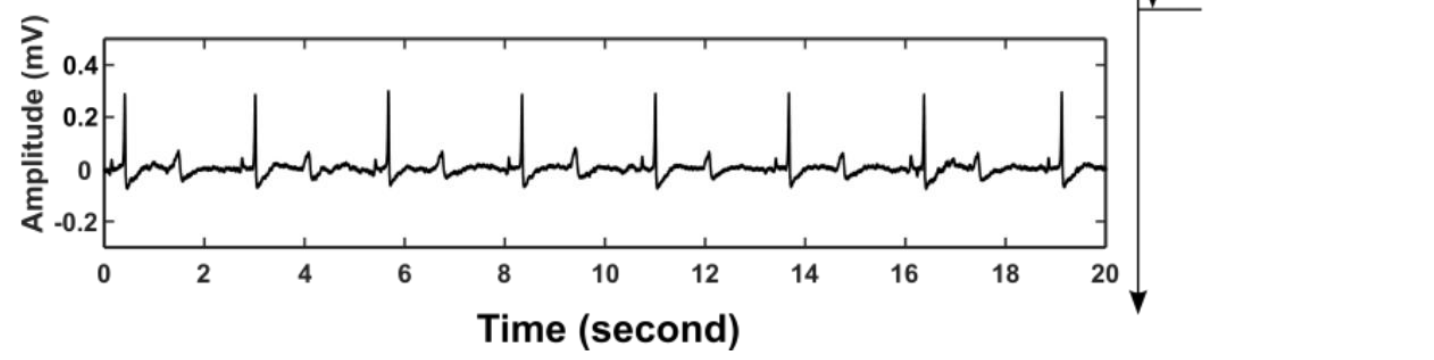

Fig. 6. Abnormal ECG found in PTZ-treated Xenopus. A. T wave gradual inversion in 